\title{
Neural stem cells, acetylcholine and Alzheimer's disease
}

\section{To the editor:}

The study by Diamandis et al. ${ }^{1}$ in your May issue reported the results of the screening of molecules that affect neurotransmission pathways in a neural stem cell model; many of these molecules had potent effects on neural stem cell proliferation. The discovery of multipotent neural stem cells within the brain has generated interest in finding ways to control their proliferation for therapeutic use. Significantly, the authors showed that neural stem cell proliferation is changed by neuromodulatory agents, suggesting that neural stem cell proliferation could be influenced, in patients, by existing clinically prescribed neuroactive drugs. This scenario opens exciting new possibilities for the treatment of neurodegenerative diseases. In particular, the finding that acetylcholinereceptor antagonists have an antiproliferative effect on neural stem cells has important implications for the understanding and treatment of Alzheimer's disease.

The brains of patients with Alzheimer's disease have reduced levels of acetylcholine ${ }^{2}$. The most successful treatment for the disease is the use of acetylcholinesterase inhibitors such as galantamine and donepezil, which effectively increase the levels of acetylcholine ${ }^{3}$. Critically, as well as reducing the symptoms of Alzheimer's disease, there is clear evidence that acetylcholinesterase inhibitors have an additional neuroprotective activity. Studies have shown that these drugs have diseasemodifying, rather than purely symptomatic, effects $^{4,5}$. Recent work has also shown that patients treated with these drugs accumulate lower levels of $\beta$-amyloid within their brains ${ }^{6}$.

The use of acetylcholinesterase inhibitors in Alzheimer's disease can be considered analogous to the use of acetylcholinereceptor antagonists in the authors ${ }^{1}$ neural stem cell model, which, they showed, results in decreased proliferation of stem and progenitor cells. This is of great significance because it is now known that neurogenesis occurs in the adult brain and that it can even occur in the brains of patients with Alzheimer's disease ${ }^{7}$, where it seems probable that it may delay the progression of the disease. The mechanism for the neuroprotective action of acetylcholinesterase inhibitors is not yet understood, but the work by Diamandis et al. ${ }^{1}$ suggests that the mechanism of action could involve increased acetylcholine levels acting on the acetylcholine receptors of neural stem cells, thus restoring or increasing the proliferative activity of these cells. Increased proliferation of neural stem cells may well mitigate or reduce disease progression.

The paper by Diamandis et al. ${ }^{1}$ opens a new angle of attack for the understanding and treatment of neurodegenerative diseases such as Alzheimer's disease, something that is desperately needed if we are to overcome this complex and intractable condition.

\section{Nigel J Fullwood}

Biomedical Sciences Unit, Department of Biological Sciences, Lancaster University, Bailrigg, Lancaster LA1 4YQ, UK. e-mail:n.fullwood@lancaster.ac.uk

1. Diamandis, P. et al. Nat. Chem. Biol. 3, 268-273 (2007).

2. Raiteri, M. Pharmacol. Rev. 58, 162-193 (2006).

3. Ginestet, L., Ferrario, J.E., Raisman-Vozari, R., Hirsch, E.C. \& Debeir, T. J. Neurochem., 102, 434-440 (2007).

4. Raskind, M.A., Peskind, E.R., Truyen, L., Kershaw, P. \& Damaraju, C.V. Arch. Neurol. 61, 252-256 (2004).

5. Allsop, D., Martin, F.L., Moore, S. \& Fullwood, N.J. Lancet 368, 361 (2006).

6. Ballard, C.G. et al. Neurol. 68, 1726-1729 (2007).

7. Jin, K. et al. Proc. Natl. Acad. Sci. USA 101, 343-347 (2004). 